\section{Cahiers de Narratologie}

Analyse et théorie narratives

17 | 2009

Stéréotype et narration littéraire

\title{
Dénonciation et récupération du stéréotype dans l'œuvre romanesque de Jardiel Poncela
}

\section{Cécile François}

\section{(2) OpenEdition}

Journals

Édition électronique

URL : http://journals.openedition.org/narratologie/1215

DOI : 10.4000/narratologie.1215

ISSN : 1765-307X

Éditeur

LIRCES

\section{Référence électronique}

Cécile François, « Dénonciation et récupération du stéréotype dans l'œuvre romanesque de Jardiel Poncela », Cahiers de Narratologie [En ligne], 17 | 2009, mis en ligne le 22 décembre 2009, consulté le 19 avril 2019. URL : http://journals.openedition.org/narratologie/1215; DOI : 10.4000/ narratologie. 1215

Ce document a été généré automatiquement le 19 avril 2019

\section{c) $(1)(-$}

Cahiers de Narratologie - Analyse et théorie narratives est mis à disposition selon les termes de la licence Creative Commons Attribution - Pas d'Utilisation Commerciale - Pas de Modification 4.0 International. 


\title{
Dénonciation et récupération du stéréotype dans l'œuvre romanesque de Jardiel Poncela
}

\author{
Cécile François
}

Dans son ouvrage intitulé Stéréotype et lecture, Jean-Louis Dufays définit le genre discursif comme « une matrice de stéréotypes » et, plus précisément, comme « un ensemble plus ou moins organisé de séquences stéréotypées [...] et de topoï qui permet de structurer une série illimitée de discours ${ }^{1} »$. D'un point de vue pragmatique, la perception générique conditionne fortement la lecture puisque le genre constitue également, selon la définition de Philippe Hamon, « un horizon d'attente thématique et formel institutionnalisé ${ }^{2}$ ». Il faut toutefois préciser que tous les architextes ne présentent pas le même degré de stéréotypie. Par ailleurs, tous les lecteurs ne ressentent pas le même agacement ou la même lassitude face à la répétition des patrons scénaristiques, des dénouements convenus, des figures imposées. Comme le remarque Isabelle Cani, certains genres mettent en place une véritable esthétique de la répétition qui, paradoxalement, redouble le plaisir du lecteur ${ }^{3}$. Il s'agit essentiellement des productions de la littérature populaire qui s'inscrivent dans une logique de diffusion de masse et s'adressent avant tout à des lecteurs envisagés comme des consommateurs de divertissement au sens le plus large.

Dans l'Espagne du début du XX ${ }^{\text {ème }}$ siècle, c'est le roman d'amour décliné sous ses différentes modalités qui suscite l'engouement du public. Si les lecteurs se ruent, chaque semaine, sur les fascicules bon marché qui leur dispensent leur lot de rêves formatés, de nombreuses voix s'élèvent pour critiquer le conformisme des représentations toutes faites, la banalité des scénarios ritualisés et le ressassement de figures usées jusqu'à la corde. S'inscrivant dans le cadre des Avant-gardes littéraires qui affichent un mépris souverain des formes établies, de jeunes auteurs font ainsi du roman d'amour la cible de leurs critiques et de leurs railleries. Parmi ceux-ci figure Enrique Jardiel Poncela qui, dans le paratexte de sa trilogie humoristique ${ }^{4}$, nous révèle explicitement ses intentions. Il s'agit, pour lui, de s'amuser aux dépens d'un certain nombre d'auteurs à succès en 
proposant à ses lecteurs une parodie du système idéologique, narratif et stylistique de la littérature amoureuse.

La lutte contre la stéréotypie

3 Lorsqu'on se penche sur les déclarations de Jardiel Poncela, on s'aperçoit que le terme qu'il emploie le plus couramment est celui de « tópico ». Il s'agit, en espagnol, d'un mot aux contours assez flous puisqu'il englobe les notions de « lieu commun » et de «cliché ", et se présente à la fois comme un « schéma formel et conceptuel ${ }^{5}$ ». La notion ainsi définie semble correspondre, au moins en partie, au terme français " poncif », dont Ruth Amossy et Anne Herschberg Pierrot nous disent qu'il « qualifie, en littérature, une thématique, un personnage ou un style convenu $»^{6}$. Par son extension sémantique très large, il recouvrirait, à la fois, le champ du stéréotype et celui du cliché et renverrait à la notion d'œuvre banale, conçue par un esprit routinier, reproduisant des thématiques et des formes conventionnelles. Envisagé sous cet aspect, le «poncif » correspondrait assez bien à ce que Jardiel Poncela désignait sous le terme de «tópico ». María José Conde Guerri, dans l'un de ses ouvrages sur le théâtre de Jardiel Poncela, signale, en effet, que «l'auteur entendait par 'tópico' tous les lieux communs diffusés par la littérature mélodramatique de l'époque qui étaient devenus de véritables stéréotypes ${ }^{7}$ ».

4 L'inconvénient de l'emploi du terme "poncif » tient à son caractère archaïque. Ruth

Amossy et Anne Herschberg Pierrot remarquent que le mot ne fait plus partie du langage critique. Il faut donc, pour rendre compte du « tópico » espagnol, se tourner vers le terme "stéréotype " envisagé dans son acception la plus large, c'est-à-dire comme "une structure verbale, thématico-narrative ou idéologique qui se signale par sa fréquence, son caractère inoriginé, son figement et le caractère problématique de sa valeur (esthétique, morale, référentielle $\left.{ }^{8}\right)$ ». Ainsi défini, le terme « stéréotype » se déclinerait dans ses deux versions, structurelle et formelle, le socle commun étant cette notion de «fixité » ou de « figement » qui donne au récepteur (ici au lecteur) un sentiment de « déjà-lu ». Dans la cadre de la satire littéraire mise en place dans la trilogie de Jardiel Poncela, le terme "stéréotype » au sens large pourrait rendre compte à la fois de la récurrence des motifs et de l'uniformité des scénarios d'une part et, d'autre part, du ressassement des figures rhétoriques et stylistiques qui caractérisent certains types de productions narratives telles que le roman d'amour.

5 Tout au long de la trilogie, le romancier espagnol se donne, en effet, pour mission de traquer le stéréotype et de débusquer le cliché en les exhibant aux yeux du lecteur. Les trois romans mettent en place un "narrateur-auteur», occupé à écrire un roman d'amour avec le souci affiché d'en respecter les paradigmes narratifs traditionnels. L'un des intérêts majeurs de l'œuvre romanesque de Jardiel Poncela étant l'importance de sa dimension métanarrative, le récit est constamment parasité par des commentaires, des jugements ou des interventions visant à justifier telle ou telle pratique. C'est ainsi que le narrateur du premier roman interrompt brusquement le récit de la rencontre des deux héros pour se livrer à un exercice de style obligé, en l'occurrence le portrait de l'héroïne. Mais, au seuil de la description, une parenthèse dévie l'attention du lecteur en référant à l'acte d'énonciation par un commentaire faussement contrit, imprimé de surcroît en italiques : «(Comment! Ne pas encore avoir décrit Lady Brums! Mais, dans un roman d'amour, c'est une erreur impardonnable et il faut la réparer tout de suite ${ }^{9}$ ).

6 Ces parabases récurrentes ont pour mission d'attirer constamment l'attention sur les codes et les conventions qui régissent la construction du roman d'amour traditionnel en jetant un regard oblique et démystifiant sur ce genre de littérature. Dans le deuxième 
volet de la trilogie, le narrateur dénonce ainsi ouvertement les scénarios routiniers du roman populaire en proposant une double lecture d'un épisode incontournable. Dans un premier temps, la scène est introduite par un commentaire métanarratif en italique qui ne laisse aucun doute sur la position de Jardiel Poncela vis-à-vis des conventions du genre: "Voici à présent comment devrait se dérouler le récit si l'auteur était un idiot». Le narrateur se livre alors à une parodie savoureuse de la séquence stéréotypée avant de communiquer au lecteur sa propre version démystifiante: «Mais comme l'auteur n'est pas encore un idiot, le récit se déroule tout autrement, de la façon suivante ${ }^{10}$ ». Le parallélisme de la construction permet ainsi au narrateur de prendre ses distances par rapport à une littérature qu'il qualifie souvent de "nauséabonde», ou de "faisandée " (l'adjectif « putrefacta » revient à maintes reprises sous sa plume). Le narrateur du troisième roman n'hésite d'ailleurs pas à exprimer ouvertement son rejet, voire son dégoût, au moment de se plier aux règles qui président à l'élaboration de son récit :

Ah! Comme j'aimerais peindre l'idylle que la naïve jeune fille amoureuse vit auprès du fringant lieutenant d'Infanterie ou du zélé licencié en Droit. Je voudrais décrire leurs tendres duos d'amour dans le jardin [...]. Mais, je le jure, je ne peux pas. Tout cela me donne la nausée ${ }^{11}$.

7 Dans les romans de Jardiel Poncela, la mise à distance du stéréotype se fait sur un mode beaucoup plus satirique qu'humoristique. Le ton est souvent railleur, sans aménité, parfois empreint d'une certaine agressivité, comme en témoigne cette citation extraite du premier roman: "'Les événements se précipitaient', comme écrivent les arriérés mentaux de la littérature ${ }^{12}$ ». Dans la trilogie, le stéréotype fait l'objet d'un jugement négatif puisqu'il est dénoncé comme un signe de faiblesse mentale et culturelle. L'humour de Jardiel Poncela apparaît ainsi comme essentiellement iconoclaste. En ce sens, la trilogie s'inscrit dans le vaste mouvement de rénovation artistique et littéraire qui caractérise les premières décennies du XX ${ }^{\text {ème }}$ siècle. L'art nouveau prôné et mis en place par les Avant-gardes se caractérise, en effet, par une volonté de rupture avec les modèles hérités de la tradition. Par ailleurs, dans son essai, intitulé La Déshumanisation de l'art ${ }^{13}$, le philosophe Ortega y Gasset fait de l'humour une des caractéristiques des nouvelles esthétiques, un humour revisité par les analyses de Bergson et de Freud. Si ce dernier parle de « l'économie d'une dépense de sentiment », Bergson, de son côté, évoque cette " amnésie momentanée du cœur » sans laquelle le rire ne peut surgir ${ }^{14}$. Pour faire éclore un nouvel art non réaliste, abstrait, esthétique, un «art artistique » selon l'expression d'Ortega y Gasset, l'écrivain (ou le peintre) doit faire appel à la mise à distance mais également procéder à une " purification de l'art» en le purgeant de tout le pathos qui encombrait déjà la littérature romantique. La trilogie de Jardiel Poncela s'inscrit ainsi dans ce courant de l'Avant-garde dont l'un des projets consistait en l'élimination du sentimentalisme grâce au pouvoir corrosif d'un nouveau concept d'humour. Comme l'écrit Jardiel Poncela dans le prologue de son premier roman : "J'ai toujours utilisé ma plume comme un insecticide ${ }^{15} »$.

8 Dans ces conditions, on comprend pourquoi la verve satirique de l'auteur s'exerce avant tout à l'encontre des stéréotypes du roman d'amour. Ce genre extrêmement codifié se construit, en effet, à partir d'une série de séquences narratives incontournables et puise dans un fonds commun de motifs et de thèmes récurrents dont usent et abusent les auteurs des fascicules destinés au grand public. Tout au long de la trilogie, Jardiel Poncela s'emploie à dénoncer le pseudo-romantisme d'une littérature larmoyante qu'il fustige. Parmi les séquences stéréotypées ou les morceaux de bravoure qui constituent les 
invariants du genre figure tout naturellement le fameux « duo d'amour » que les lectrices attendaient toujours avec la plus grande impatience.

La subversion d'une séquence narrativo-thématique

9 Dans le roman sentimental, la scène d'amour comporte une série de motifs obligés qui sont devenus au fil du temps de véritables poncifs. C'est ainsi que les étoiles, la lune, les parfums du soir, la brise nocturne constituent pour le héros une source d'inspiration dans laquelle il puise abondamment pour déclarer sa flamme à l'élue de son coeur. Le narrateur du premier volet de la trilogie est d'ailleurs parfaitement conscient de l'aubaine que constitue pour le romancier le vaste catalogue de clichés et de stéréotypes grâce auquel il peut suppléer son manque d'imagination: "(Quelle chance pour les romanciers lorsque leurs personnages se mettent à contempler la lune. Si la lune n'existait pas, beaucoup de romans n'auraient pas pu être écrits, pas même celui-ci ${ }^{16}$ )».

Cette remarque glissée au détour d'un paragraphe confirme l'intention démystificatrice de Jardiel Poncela. En effet, si le narrateur laisse entendre à la fin de la citation que sa propre narration n'échappe pas aux pièges de la stéréotypie, le lecteur saisit immédiatement la charge ironique de ses paroles. Dès le paratexte, l'auteur a été on ne peut plus explicite. Ce qu'il propose au lecteur, c'est une satire littéraire, c'est-à-dire, un récit au second degré, ou selon la définition de Maria Grazia Profeti, «une métalittérature, une modalité qui prospère aux dépens d'une autre réalité littéraire ${ }^{17}$ ». L'autocritique du narrateur est donc, bien évidemment, feinte puisqu'il s'agit moins, pour lui, d'imiter un modèle que de s'en écarter, en puisant dans l'arsenal pseudo-romantique tous les stéréotypes susceptibles d'être démontés, déconstruits, subvertis par le biais de son humour caustique. On peut voir que Jardiel Poncela rejoint ici, avec un peu moins d'agressivité toutefois, le mot d'ordre que Marinetti, le chef de file des futuristes, avait lancé dès 1909 : « Tuons le clair de lune ! ».

11 C'est ainsi que, dans le premier roman de la trilogie, un train emporte vers Paris un couple qui contemple la nuit étoilée par la fenêtre du compartiment. Emu par la beauté du paysage nocturne, le protagoniste ne peut s'empêcher de manifester ses sentiments et de s'exclamer en se tournant vers sa compagne : «J'ai toujours rêvé d'embrasser ma bienaimée au clair de lune». Mais, ici, la banalité du propos suscite immédiatement une réaction de condescendance, voire de mépris, de la part du narrateur qui juge le héros victime de cette « imbécillité monumentale propre à tant d'amoureux ». Lorsqu'il ne subit pas le dédain du narrateur, le protagoniste naïf et romanesque se heurte à l'indifférence glaciale de sa compagne. "Regarde la lune, comme elle est belle!», déclare avec enthousiasme le héros sentimental. Mais ce dernier sollicite inutilement une partenaire insensible à la beauté et à la poésie de l'instant. En effet, jouant sur la polysémie du terme espagnol «luna» [« lune » / « miroir »], l'héroïne répond très prosaïquement : « Oui. On dirait un miroir de salle de bain ${ }^{18} »$. On assiste ici à la mise en place d'un procédé humoristique que Jardiel Poncela exploite de façon récurrente tout au long de la trilogie. Il s'agit, pour l'auteur, de créer un effet de surprise amusée chez le lecteur par la désacralisation d'objets traditionnellement investis d'une fonction poétique. En assimilant la lune à un miroir de salle de bain ou à une torche électrique comme il le fera quelques lignes plus loin, Jardiel Poncela dénature par le biais d'une comparaison incongrue un objet poétique. Ce faisant, il provoque également un décalage, une rupture propice à l'émergence du rire, car selon le philosophe Bergson : « [On obtiendra] un effet amusant quand on développera un symbole ou un emblème dans le sens de leur 
matérialité et qu'on affectera de conserver à ce développement la même valeur symbolique que l'emblème ${ }^{19}$. ».

12 Selon le même procédé, la référence aux étoiles sera à son tour ridiculisée par le don Juan matérialiste et jouisseur du deuxième volet de la trilogie. Dans ¿Pero...hubo alguna vez once mil vírgenes?, le firmament, en effet, n'inspire guère d'enthousiasme ni d'envolées lyriques au protagoniste. Lorsque la tendre amoureuse lui murmure à l'oreille: "Explique-moi les étoiles, mon chéri...", celui-ci récite: "Une sur la manche, souslieutenant; deux, lieutenant; trois, capitaine ${ }^{20} \ldots$ » Dans ce cas, l'humour naît du télescopage de deux isotopies antagoniques. A l'élévation de l'âme, au désir de transcendance et de poésie de la jeune fille naïve et sentimentale, le don Juan oppose la matérialité et la rigidité de la hiérarchie militaire. Cette dégradation du symbole provoque un retour brutal à la réalité (accompagné virtuellement d'un mouvement descendant qui va du firmament à l'uniforme de l'officier).

13 La verve satirique de Jardiel Poncela s'exerce à l'encontre d'un autre motif traditionnel, celui des larmes de l'héroïne. Dans le troisième volet de la trilogie, ce moment d'intense émotion laisse ainsi la place à une scène grotesque qui met en présence deux personnages sans passion ni sensibilité. Ces âmes routinières considèrent, en effet, les larmes, non comme l'expression d'un trouble ou d'un sentiment puissant mais comme un exercice hygiénique, une sorte d'entraînement ou d'activité programmée à l'avance. C'est ainsi que lors de la promenade au clair de lune, le protagoniste propose : « Veux-tu que j'arrête la voiture et que nous pleurions dix ou douze minutes, Vivola?». Mais l'amoureuse décline l'offre de son compagnon sur un ton naturel et très détaché en répliquant: « Moi, je pleure de quatre heures à six, parce que, à sept heures, arrive la manucure ${ }^{21}$. "

14 A la différence de ce roman, le deuxième volet de la trilogie, met en scène une héroïne éperdue d'amour, prête à tout pour obtenir les faveurs du héros. Or, au moment où elle comprend que tout espoir est perdu et que rien ne pourra empêcher celui qu'elle aime d'épouser une autre femme, Palmera, la protagoniste, le laisse partir « avec la plus grande angoisse au fond du cœur, mais sans pleurer ». C'est alors que le narrateur s'interroge : "Était-ce parce que, comme pour le personnage de Sem Benelli, dans ses yeux, elle n'avait plus de larmes? Non. Ce n'était pas parce qu'elle n'avait plus de larmes dans les yeux. C'est parce qu'elle avait du rimmel. Et on sait bien que les larmes sont une vraie catastrophe pour une femme qui a du rimmel sur les yeux ${ }^{22}$. » Le recours à l'italique permet de mettre en relief le petit jeu du narrateur qui s'amuse ici à pulvériser un poncif littéraire en le remplaçant par un préjugé.

15 Dans ce même roman, on assiste, de surcroit, à un renversement de situation puisque c'est le barbon ridicule qui porte la charge émotionnelle de la scène. Le soupirant de Palmera est, en effet, un vieillard lascif et grotesque qui ne cesse, depuis le début du récit, de sangloter et de hoqueter sans raison. Ses multiples apparitions dans la fiction sont ponctuées d'une onomatopée, d'un « hi, hi, Hiiiiiiiii ! hihi, hi!», plus ou moins long, plus ou moins soutenu, qui renforce le comique de répétition et donne au personnage l'apparence d'un pantin, d'une mécanique qui se mettrait en marche automatiquement, à la moindre contrariété. C'est ce personnage ridicule qui vient s'immiscer dans le couple formé par Palmera et Mario à la fin du roman. Or, la présence de ce barbon grotesque qui ne cesse de gémir et de sangloter transforme le duo d'amour en trio comique. On remarque toutefois que, si Jardiel Poncela s'amuse aux dépens du vieux marquis dont il transforme les pleurs en hululements, les amants ne sont évidemment pas à l'abri des effets corrosifs de son humour. Et leurs tendres effusions se réduisent à une succession de baisers 
sonores dont le caractère mécanique est souligné par le recours à l'onomatopée : "schmack, schmack, schmack, schmack». Mêlant les gémissements du barbon aux baisers bruyants des amants, Jardiel Poncela tire les meilleurs effets comiques d'un polylogue cacophonique qui donne à la scène des allures de basse-cour :

Le marquis : - ¡Hi, Hi ! Hiiiiiiii ! Hi, hi !...

Palmera : - Schmack, schmack, schmack! (Trois baisers)

Mario : - Schmack, schmack, schmack, schmack! (Quatre baisers)

Le marquis : - ¡Bouuuuuuuh! (Cent larmes ${ }^{23}$ ).

Comme on le voit, c'est contre toute une littérature "préfabriquée ", encombrée de stéréotypes, de clichés, de lieux communs éculés que Jardiel Poncela ouvre les hostilités. Iconoclaste, le jeune auteur l'est donc sans aucun doute, à l'exemple des Futuristes ou même des Dadaístes. Sa pratique de la parodie générique démontre assez sa volonté de remettre systématiquement en cause un certain type de littérature à stéréotypes. Mais cette démarche critique, très souvent dévastatrice, se veut également constructive. Comme le rappelle Aline Geyssant: «La parodie vise souvent les excès des genres littéraires trop figés et, par ce biais, elle témoigne d'une volonté de renouvellement face à un ordre qui a cessé d'être créatif ${ }^{24}$.» Ainsi, au fil des pages de la trilogie, un texte original émerge et se forge à partir de la déconstruction de modèles antérieurs. La subversion des stéréotypes devient, pour Jardiel Poncela, un mode privilégié d'expression et de rénovation du genre romanesque et contribue puissamment à l'élaboration de sa propre théorie esthétique.

Des stéréotypes revisités et revitalisés

Pour revivifier et redynamiser un genre en perte de vitesse ou sur la voie du figement et de la sclérose, Jardiel Poncela se tourne vers les nouvelles techniques et les nouveaux moyens d'expression que l'époque met à sa disposition. C'est ainsi que les onomatopées et les jeux sur la disposition graphique de la page et sur la ponctuation qu'il affectionne renvoient directement à l'univers de la bande dessinée. L'épilogue du duo d'amour de Vivola et de don Pedro dans le troisième volet de la trilogie est, à cet égard, tout à fait révélateur. Refusant de se plier aux codes de la littérature populaire, le narrateur ne prend pas la peine de décrire la pose alanguie de l'héroïne. Il ne fait pas davantage état de son émotion. A la place, il nous offre le dessin d'une bouche sur laquelle s'inscrivent les mots suivants: "Trois minutes de baiser». Ces trois minutes d'ivresse ineffable sont suffisantes pour faire perdre au héros, non pas le contrôle de lui-même, mais celui de son véhicule. Les deux protagonistes terminent ainsi leur duo d'amour dans le fossé, dépités et confus, sous le regard ironique du narrateur qui met un point d'orgue à la scène par un commentaire malicieux glissé entre parenthèses: "(Cela faisait un moment que nous le redoutions car la 'Lincoln' zigzaguait dangereusement ${ }^{25}$ )». Pour renforcer le côté comique de l'épisode, Jardiel Poncela a recours aux onomatopées qui donnent à la scène d'amour des allures de bande dessinée ou de dessin animé. C'est ainsi qu'un « ¡iPlaff!! » retentissant clôt la séquence. Ce mot nettement détaché du texte imite le bruit de la voiture versant dans le fossé et ôte par là même à la scène tout son sérieux. Et ce, d'autant plus qu'il est suivi d'une seconde onomatopée («Ay») qui occupe une place démesurée dans la composition de la page. Le narrateur intervient d'ailleurs pour justifier la taille gigantesque de l'interjection « [Vivola] poussa un cri [...], un cri si grand, si grand qu'il va occuper la moitié de la page ${ }^{26} »$.

Toutefois, en ce début du XXe siècle, plus que la bande dessinée, c'est le cinéma qui est considéré comme l'invention la plus extraordinaire qui soit. Or, les grands comiques du 
burlesque partagent avec l'auteur de la trilogie le goût de la subversion des conventions, du détournement des stéréotypes, de l'inversion des situations. "Conscients de la relativité de tout, - écrit Petr Král - les comiques renversent avec joie les valeurs établies et érigées en idoles ${ }^{27}$ ». On ne s'étonnera pas, dans ces conditions, de voir Jardiel Poncela puiser abondamment dans le fonds du burlesque cinématographique, destructeur lui aussi des conventions dominantes qui régissent les genres ${ }^{28}$.

Dans le premier volet de la trilogie, Jardiel Poncela met en scène une jeune amoureuse, Ramona, dont le lecteur sait peu de choses, excepté que le trait le plus caractéristique de sa personnalité semble être une propension à la rêverie et à l'idéalisme. Son prénom est, en effet, systématiquement suivi d'une apposition qui a valeur d'antonomase: « la femme romantique ». La scène de la première rencontre du héros et de Ramona a lieu précisément dans un cinéma. La projection a déjà commencé lorsque le protagoniste arrive. C'est pourquoi, dérouté par l'obscurité de la salle, celui-ci se trompe de siège et s'assied sur Ramona, sans chercher à s'excuser ni d'ailleurs à se déplacer. On songe à cette instrumentalisation de la femme, caractéristique des films de Chaplin, puisque le héros utilise ici Ramona comme un fauteuil, de la même façon que Charlot se sert du corps d'une opulente mère de famille comme d'une passerelle pour embarquer sur le bateau du film Une Journée de plaisir tourné en 1919. Le rire, né de ce comique de situation, se prolonge lorsque Ramona, au lieu de s'indigner, accepte son rôle de victime avec une belle indifférence. «La dame ne protesta pas; elle se contenta d'écarter légèrement ma tête ", raconte le héros. Or, cette impassibilité est, on le sait, une des caractéristiques du personnage burlesque.

Puis, rapidement, la scène prend des résonances de farce énorme lorsque le fauteuil se brise et engloutit le corps de Ramona: «Le fauteuil de ma voisine se brisa et Ramona s'enfonça dans le trou du siège. Elle cria, elle hurla, une vingtaine d'employés accoururent ». La force comique des gags qui se succèdent en série est alors accentuée par le récit lui-même qui joue des ressources de l'hyperbole. L'incident se transforme en cataclysme, comme le suggère la métaphore choisie par le héros : « Nous ressemblions à deux mineurs luttant dans les ténèbres de la mine pour éviter une explosion de grisou ». Par ailleurs, le déploiement du champ lexical du naufrage évoque également le drame du Titanic, où la scène déchirante des adieux est traitée sur le mode à la fois parodique et burlesque: «les jambes de la dame s'agitaient dans l'air comme pour dire au revoir à quelqu'un ${ }^{29}$ ». Paquebot en péril ou baleine échouée sur la grève, Ramona, «la femme romantique ", épuise les efforts de ses treize sauveteurs qui ne parviendront qu'à grandpeine, au prix d'un travail surhumain, à l'extraire de son fauteuil. Aussi, bien loin de l'image éthérée de l'héroïne frêle et gracile du roman sentimental ou de la littérature romantique dont elle ne cesse de se réclamer, Ramona est présentée tout au long du récit comme un personnage éminemment comique. Et la séquence de la première rencontre est traitée sur le mode burlesque à la façon des slapsticks du cinéma des premiers temps.

Stéréotypes et effets de lecture

21 Il est évident que le traitement des stéréotypes induit un certain nombre d'effets de lecture. Leur mise à distance permanente vise à déstabiliser le lecteur en le soumettant à de brusques arrêts ou en bloquant le processus d'identification et d'absorption dans le monde diégétique. La stratégie mise en place tout au long de la trilogie vise à stimuler la réflexion du lecteur en l'amenant à adopter une attitude critique face au " prêt-à-écrire " de la littérature de masse. Ce que Jardiel Poncela cherche à établir, par le biais des innombrables commentaires métanarratifs ou métafictionnels, c'est un climat de 
connivence sans lequel le discours humoristique ne saurait fonctionner. Tout au long d'une promenade démystifiante dans les coulisses du récit, le narrateur de la trilogie invite le lecteur à réagir, non seulement en pratiquant une lecture distanciée mais également en participant activement au démontage des clichés et des stéréotypes. Car l'activité ludique qui se déploie dans l'espace des romans n'est en aucun cas un jeu solitaire du narrateur-auteur. Au contraire, le texte programme la figure d'un « lecteurcomplice ", actif et compétent, capable d'accepter de se livrer aux différentes activités qui lui sont proposées.

C'est ainsi que dans le deuxième roman, Jardiel Poncela édicte les règles d'un jeu qui prend sa source dans le discours amoureux dont l'auteur déplore l'indigence et la banalité. Le duo d'amour codifié, stéréotypé, est devenu un passage obligé si commun, si éculé, qu'on peut, sans risque, le compléter les yeux fermés. C'est pourquoi Jardiel Poncela s'amuse à transformer le dialogue des deux protagonistes en une sorte d'énigme, de jeu verbal dont il invite le lecteur à reconstituer le sens :

Palmera :- ¡V.d.!

Mario : - ¡Gl.r.. !

Palmera : - ¡C.r.z.n m.. !

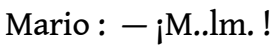

La consternation du lecteur n'est que de courte durée puisque « l'énigme » s'accompagne d'une sorte de mode d'emploi, ou plus exactement d'une méthode de décryptage fournie par l'auteur. Cette brève séquence s'intitule en effet: "Rencontre avec fugue de voyelles ", titre susceptible d'orienter le lecteur perspicace dans son déchiffrement du dialogue. En outre, afin de ne laisser subsister aucun doute, une note de bas de page vient donner quelques indications supplémentaires sur la marche à suivre, sans pour autant fournir une transcription directe des paroles des deux personnages : "remettez à leur place les voyelles suivantes : i, a, o, i, a, o, a, ó, í, o, i, a, a ${ }^{30}$. »

Outre le jeu de "l'exercice à trous ", la devinette permet également à l'auteur de pointer $\mathrm{du}$ doigt nos habitudes de lecture pour nous obliger à adopter une attitude de distanciation critique à l'égard du stéréotype. Dans le dernier volet de la trilogie, la séquence du duo d'amour s'achève, comme on le sait, sur une note mi-dramatique, miburlesque lorsque la voiture des deux protagonistes verse dans le fossé. En fait, le narrateur ne se contente pas de traiter les conséquences de l'accident en empruntant aux codes de la bande dessinée. Il va plus loin en proposant une sorte de devinette qui tire le dénouement de la séquence vers le domaine de l'incongruité et de l'absurde. Commentant la réaction de l'héroïne, le narrateur écrit en effet: «Elle poussa un hurlement (pour montrer son affolement? Pour prévenir Pedro? Pour effrayer la voiture? Ou pour attendrir le bascôté? On n'a jamais pu le savoir ${ }^{31}$ )». Ici, l'activité du lecteur n'est pas directement sollicitée. Il ne s'agit pas, pour lui, d'exercer sa compétence linguistique mais de faire preuve d'humour en sachant apprécier à sa juste valeur une avalanche de questions ou d'hypothèses plus saugrenues les unes que les autres, destinée non seulement à le faire sourire mais également à rompre le déterminisme de la lecture.

L'humour de Jardiel Poncela se fonde en effet sur la notion d'incongruité qui peut par moments déboucher sur le «nonsense ». Albert Laffay utilise, pour le définir, l'image "d'une machine à parler qui s'emballe ou plutôt qu'on laisse, par moments, s'emballer ». «Le nonsense - écrit-il -, c'est l'humour lorsqu'on le dirige vers l'utilisation déréglée, ou du moins l'emploi autonome, du langage ${ }^{32} »$. Jardiel Poncela y a recours pour subvertir le 
motif de la passion dévorante qui fait tourner la tête de l'héroïne ou aliène sa raison. Dans le deuxième roman, le narrateur révèle ainsi au lecteur les sentiments qu'éprouve Palmera à l'égard de Mario par le biais d'une série d'anadiploses. Or, cet enchaînement, apparemment logique sur le plan syntaxique, débouche sur une aberration sémantique :

Elle était amoureuse, très amoureuse, éperdument amoureuse, amoureuse à la folie, de Mario Esfarcies. Et Mario Esfarcies méritait cet amour même s'il ignorait son existence. Et il ignorait son existence même s'il habitait un étage au-dessus de celui de Palmera. Et il habitait un étage au-dessus même s'il allait se marier. Et il allait se marier, même s'il avait les cheveux bruns ${ }^{33}$.

On remarque que, même si l'hyperbole destinée à traduire l'intensité de la passion peut faire sourire, la première phrase reste cependant cohérente. Peu à peu cependant la logique commence à se disloquer sous l'effet de la concaténation. Le discours s'emballe et se met alors à tourner à vide comme dans ces jeux d'enfants dont certains consistent à enchaîner la dernière syllabe d'un mot à la première du mot suivant et dont l'exemple type est le fameux " marabout / bout de ficelle ».

Ce que la trilogie dessine en filigrane, ce n'est donc pas seulement la figure d'un lecteur capable idéalement de repérer les stéréotypes et de prendre plaisir à la façon dont le narrateur les met à distance pour mieux les subvertir, ce qui est sans doute le propre de tout amateur de discours humoristique. Ce que Jardiel Poncela attend et espère de son public, c'est qu'il soit amené à changer ses habitudes de lecture et à passer du rôle de « lisant » à celui de « lectant », selon la terminologie forgée par Vincent Jouve ${ }^{34}$. L'image du lecteur que programme le texte de la trilogie est celle d'une instance intellectuelle capable d'exercer en permanence sa réflexion critique et de pratiquer une lecture "déshumanisée », c'est-à-dire désinvestie de son lot habituel d'affects et d'émotions diverses. Il s'agit également pour l'auteur d'initier son lecteur à une nouvelle forme d'humour qui s'enracine dans le terreau des Avant-gardes, dont les mots d'ordre étaient «rupture » et "nouveauté ». L'art nouveau fondamentalement iconoclaste, ludique et anti-sentimental revendique, en effet, cette insouciance et cette légèreté caractéristiques des «Années folles» en plaçant le jeu, l'humour et la frivolité au coeur de l'oeuvre littéraire et artistique. Le but, pour les artistes de l'Avant-garde, était de faire table rase du passé, de délester l'art de son sérieux en le purgeant de ses aspects sentimentaux et pathétiques, de rejeter l'héritage "réaliste", en proposant un art conçu comme une activité ludique autonome, dégagée de toute problématique historique, sociale ou politique.

Conclusion

Rédigés dans l'effervescence culturelle et artistique des années 20, les romans de Jardiel Poncela portent la marque de leur époque. Tout au long de la trilogie, l'auteur se donne, en effet, pour mission de traquer les stéréotypes de toute nature (narratifs, thématiques ou stylistiques) en les exhibant aux yeux du lecteur avant de procéder à leur démontage dans une pratique ludique du récit, du langage et de l'écriture pleinement consciente d'elle-même. Se définissant avant tout comme un humoriste, le jeune romancier madrilène était déjà, à cette époque, en contact avec un groupe d'écrivains et de dessinateurs qui avaient pour noms José López Rubio, Edgar Neville, Tono, ou encore Miguel Mihura. Ensemble, ils avaient conçu et mis au point une nouvelle forme d'humour iconoclaste basé, en particulier, sur la subversion des clichés, le détournement des formules figées, le démontage des conventions linguistiques. «Il ne faut laisser passer aucun lieu commun, aucune phrase toute faite, aucun poncif ${ }^{35}$ ", tel était le mot d'ordre de ce groupe de jeunes humoristes qui allaient bientôt former ce que l'on appellerait 
"l'Autre Génération de $27^{36}$ ». Dans la trilogie, Jardiel Poncela poursuit cette œuvre de déconstruction $\mathrm{du}$ "prêt-à-parler » et du "prêt-à-écrire » commencée au début de la décennie dans les rédactions des deux grandes revues humoristiques de l'époque, Buen Humor et Gutiérrez. Mais, désormais, sa verve s'exerce plus précisément à l'encontre des écrivains qui bâtissent leurs oeuvres à grands coups de clichés et de stéréotypes, à destination d'un public plus consommateur que lecteur. Dans sa lutte contre la littérature "préfabriquée », Jardiel Poncela pointe du doigt les situations conventionnelles, les motifs récurrents, les dénouements convenus. Mais il ne se contente pas de déconstruire ou de démonter les stéréotypes. En allant chercher dans la bande dessinée ou le cinéma les instruments qui lui permettent de dynamiter des pratiques romanesques dépassées, l'auteur de la trilogie revitalise des séquences et des motifs figés. Le geste iconoclaste et subversif se transforme en pratique constructive qui lui permet de forger un nouveau concept de littérature humoristique et de s'inscrire dans le vaste mouvement de rénovation de l'écriture romanesque qui est au cœur des préoccupations des écrivains de l'Avant-garde.

\section{BIBLIOGRAPHIE}

AMOSSY Ruth et HERSCHBERG PIERROT Anne, Stéréotypes et clichés, Paris, Nathan, 1997.

AMOSSY Ruth et ROSEN Elisheva, Les Discours du cliché, Paris, CDU et SEDES réunis, 1982.

ANSCOMBRE Jean-Claude (dir.), Théorie des topoï, Paris, Kimé, 1995.

BERGSON Henri, Le Rire [1900], Paris, P.U.F., 1969.

CANI Isabelle, « Comment émergent les stéréotypes ? Le cas de l'auto-guérison à travers trois genres littéraires », Loxias 17, Actes de la journée d'études Littérature à stéréotypes, Nice, 23 février 2007.

CONDE-GUERRI María José, El teatro de Enrique Jardiel Poncela : aproximación crítica, Zaragoza, Institución Fernando el Católico, 1981.

DUFAYS Jean-Louis, Stéréotype et lecture, Liège, Mardara, 1994.

DUFAYS Jean-Louis, « Stéréotypes, lecture littéraire et postmodernisme », in Christian Plantin

(dir.), Lieux communs, topoï, stéréotypes, clichés, Paris, Kimé, 1993.

FREUD Sigmund, Le Mot d'esprit et sa relation à l'inconscient [1905], Paris, Gallimard, NRF, 1988.

FRANÇOIS Cécile, Enrique Jardiel Poncela et la rénovation de l'écriture romanesque à la fin des années 20, A.N.R.T., 2005 .

GeysSAnT Aline, GUTEVILle Nicole et RAZACK Asifa, Le Comique, Paris, Ellipses, 2000.

HAMON Philippe, « Clausules », Poétique 24, Seuil, 1975.

Cahiers de Narratologie, 17 | 2009 
JOUVE Vincent, L'Effet personnage dans le roman, Paris, PUF, 1992.

KRÁL Petr, Le Burlesque ou Morale de la tarte à la crème, Paris, Stock, 1986.

LAFFAY Albert, Anatomie de l'humour et du nonsense, Paris, Masson et Cie, 1970.

MARIE Michel, « Muet », Lectures du film, Paris, Albatros, 1975.

ORTEGA Y GASSET José, La deshumanización del arte y otros ensayos de crítica [1925], Madrid, Espasa

Calpe, 1997.

JARDIEL PONCELA Enrique, Amor se escribe sin hache [1928], Edition de Roberto Pérez, Madrid, Cátedra, 1990.

JARDIEL PONCELA, Enrique, ¡Espérame en Siberia, vida mía! [1929], Edition de Roberto Pérez, Madrid, Cátedra, 1992.

JARDIEL PONCELA Enrique, Pero... ¿hubo alguna vez once mil vírgenes? [1930], Edition de Luis Alemany, Madrid, Cátedra, 1988.

PLANTIN Christian (dir.), Lieux communs, topoï, stéréotypes, clichés, Paris, Kimé, 1993.

PROFETI Maria Grazia, « Código ideológico-social, medios y modos de la risa en la comedia del siglo XVII ", in Risa y sociedad en el teatro español del Siglo de Oro, Actes du $3^{\text {ème }}$ colloque du Groupe d'Etudes Sur le Théâtre Espagnol, Ed.du C.N.R.S., Toulouse, 31 janvier-2 février 1980.

\section{NOTES}

1. Jean-Louis Dufays, Stéréotype et lecture, Liège, Mardara, 1994, p.92.

2. Philippe Hamon, «Clausules », Poétique 24, Seuil, 1975, p.500.

3. Isabelle Cani, «Comment émergent les stéréotypes? Le cas de l'auto-guérison à travers trois genres littéraires ", Loxias 17, Actes de la journée d'études Littérature à stéréotypes, Nice, 23 février 2007.

4. Il s'agit de Amor se escribe sin hache [Amour s'écrit sans h] [1928], Madrid, Cátedra, 1990, ¡Espérame en Siberia, vida mía! [Attends-moi en Sibérie, ma chérie] [1929], Madrid, Cátedra, 1992 et de Pero... ¿hubo alguna vez once mil vírgenes? [Mais y a-t-il jamais eu onze mille vierges?] [1930], Madrid, Cátedra, 1988. Aucun de ces romans n'a été publié en français.

5. La définition du Dictionnaire de l'Académie espagnole est la suivante : « Lugar común que la retórica antigua convirtió en fórmulas o clichés viejos y admitidos en esquemas formales o conceptuales de que se sirvieron los escritores con frecuencia. » 6. Ruth Amossy et Anne Herschberg Pierrot citent l'édition de 1874 du dictionnaire Larousse: « On a donné par extension en littérature, comme en peinture et en sculpture, le nom de poncif aux compositions qui manquent d'originalité et qui semblent faites sur un patron commun ", Stéréotypes et clichés, Paris, Nathan, 1997, p.14.

7. María José Conde-Guerri, El teatro de Enrique Jardiel Poncela: aproximación crítica [Le Théâtre de Enrique Jardiel Poncela : approche critique], Zaragoza, Institución Fernando el Católico, 1981, p.43.

8. Jean-Louis Dufays. « Stéréotypes, lecture littéraire et postmodernisme », in Christian Plantin (dir.), Lieux communs, topoï, stéréotypes, clichés, Paris, Kimé, 1993, p.80.

9. « (jParece mentira! ¡No haber descrito todavía a lady Brums! Esto, en una novela de amor, es un defecto imperdonable y hay que corregirlo al punto.) ", Amor se escribe sin hache, éd.cit., p.161. 
10. « He aquí cómo tendría que desarrollarse ahora la narración si el autor fuera un idiota. [...] Pero como el autor todavía no es idiota, la narración se desarrolla de esta distinta manera » ¡Espérame en Siberia, vida mía!, éd.cit., p. 299.

11. « ¡Ay, yo bien querría pintar el idilio que la ingenua muchacha enamorada sostiene con el gallardo teniente de Infantería o con el estudioso licenciado en derecho!... Querría describir sus tiernas escenas de amor en el jardín [...]. Mas - lo juro - : no puedo. Todo eso me da náuseas. » Pero... ¿hubo alguna vez once mil vírgenes?, éd.cit., p.73.

12. «'Los acontecimientos se precipitaban', como escriben los retrasados mentales de la literatura ", Amor se escribe sin hache, éd.cit., p.193.

13. José Ortega y Gasset, La deshumanización del arte y otros ensayos de crítica [La Déshumanisation de l'art et autres essais critiques] [1925], Madrid, Espasa Calpe, 1997.

14. Sigmund Freud, Le Mot d'esprit et sa relation à l'inconscient [1905], Paris, Gallimard, NRF, 1988, p.419 et Henri Bergson, Le Rire [1900], Paris, P.U.F., 1969, p.4.

15. «Siempre empleé la pluma como un insecticida », Amor se escribe sin hache, éd.cit., p.75.

16. "¡Qué gran recurso para los novelistas es que los personajes se pongan a contemplar la luna! Si la luna no existiese, muchas novelas no se habrían podido escribir: ni siquiera ésta... », Amor se escribe sin hache, éd. cit., p.236.

17. « una meta-literaturadad, una modalidad que crece a costa de otra realidad literaria », Maria Grazia Profeti, « Código ideológico-social, medios y modos de la risa en la comedia del siglo XVII ", in Risa y sociedad en el teatro español del Siglo de Oro, Actes du $3{ }^{\text {ème }}$ colloque du Groupe d'Etudes Sur le Théâtre Espagnol, Ed.du C.N.R.S., Toulouse, 31 janvier-2 février 1980, p.17.

18. « Siempre he soñado con besar a la mujer amada a la luz de la luna - prosiguió él con esa imbecilidad astronómica propia de tantos enamorados. [...] Mira la luna, ¡qué hermosa está!... / Sí. Parece un espejo de cuarto de baño - comentó Sylvia displicentemente. » Amor se escribe sin hache, éd.cit., p.222.

19. Henri Bergson, op.cit., p.89.

20. « Explícame las estrellas, mon chéri... / El contestó con aire de antiguo miliciano: / Una sobre la bocamanga, alférez; dos, teniente; tres, capitán. » Pero... ¿hubo alguna vez once mil vírgenes?, éd. cit., p.444.

21. « ¿Quieres que pare el coche y lloremos diez o doce minutos, Vivola? / Y [don Pedro] confesó: 'Yo lloro mucho. Lloro todos los días: de cinco a siete.' / 'También yo lloro declaró ella-. Pero yo lloro de cuatro a seis, porque a las siete viene la manicura.' » Pero... ¿hubo alguna vez once mil vírgenes?, éd. cit., p.181.

22. «Palmera lo vio irse con la máxima angustia en el alma pero sin llorar. ¿Era que, como el personaje de Sem Benelli, en sus ojos ya no tenía lágrimas? No. No era que no tuviese lágrimas en sus ojos. Era que tenía rimmel. Y ya se sabe qué catástrofe constituye el llanto para una mujer que tiene rimmel en los ojos. " ¡Espérame en Siberia, vida mía!, éd.cit., p.131. 23. « El marqués: -jJí, jí! Jiiiiiiií! Ji, jí!... / Palmera: - Chás, chás, chás! (Tres besos) / Mario: ¡Chás, chás, chás, chás! (Cuatro besos) / El marqués: - ¡Uuuuuuúh! (Cien lágrimas) » ¡Espérame en Siberia, vida mía!, éd.cit., p.370.

24. Aline Geyssant, Nicole Guteville et Asifa Razack, Le Comique, Paris, Ellipses, 2000, p.54. 25. « (Hacía rato que nos lo estábamos temiendo, porque el 'Lincoln' iba trazando unas eses terribles.) », Pero... ¿hubo alguna vez once mil vírgenes?, éd.cit., p.185.

26. « [Vivola] emitió un alarido. [...] Pero emitió un alarido tan grande, tan grande, que va a ocupar media página ", Pero... ¿hubo alguna vez once mil vírgenes?, éd.cit., p.185 (page reproduite en annexe). 
27. Petr Král, Le Burlesque ou Morale de la tarte à la crème, Paris, Stock, 1986, p.231.

28. Michel Marie, « Muet », Lectures du film, Paris, Albatros, 1975, p.169.

29. «La señora no protestó; limitóse a ladearme un poco la cabeza. [...] la butaca de mi vecina se rompió y Ramona se hundió por el agujero del asiento. Gritó, aulló, acudieron doce acomodadores [...]. Parecíamos dos mineros bregando en las tinieblas de la mina para librarnos de una explosión de gas grisú. [...] las piernas de la dama se agitaban en la atmósfera como si dijeran adiós a alguien. » Amor se escribe sin hache, éd.cit., p.145-146. 30. « Encuentro con fuga de vocales / Palmera : ¡ ¡.d.! / Mario :- ¡Gl.r..! / Palmera :- ¡C.r.z.n m..! / Mario : - ¡M..lm.! / "Colóquense en su sitio la vocales i, a, o, i, a, o, a, ó, í, o, i, a, a. / Ce qui donne : « Palmera : ¡ ¡Vida! / Mario : ¡ Gloria! / Palmera :- ¡Corazón mío! / Mario : - ¡Mi alma! » ¡spérame en Siberia, vida mía!, éd.cit., p.370.

31. «Ella emitió un alarido. (¿Para demostrar pánico? ¿Para advertir a Pedro? ¿Para asustar al coche? ¿O para ablandar a la cuneta? Nunca pudo saberse.) ", Pero... ¿hubo alguna vez once mil vírgenes?, éd.cit., p.185.

32. Albert Laffay, Anatomie de l'humour et du nonsense, Paris, Masson et Cie, 1970, p.118 et 153.

33. «Estaba enamorada, enamoradísima, enamorada locamente, enamorada hasta la dislocación de Mario Esfarcies. Y Mario Esfarcies se merecía aquel amor, aunque ignoraba su existencia. E ignoraba su existencia, aunque vivía en el piso superior al de Palmera. Y vivía en el piso superior, aunque iba a casarse. E iba a casarse, aunque era moreno. » ¡Espérame en Siberia, vida mía!, éd.cit., p.116.

34. Vincent Jouve, L'Effet personnage dans le roman, Paris, PUF, 1992, p.81 à 89.

35. « No hay que dejar pasar ningún lugar común, ninguna frase hecha, ningún tópico ", cité par Herminio Pérez Fernández, « El teatro de Tono », in El teatro de humor en España [Le Théâtre humoristique en Espagne], Madrid, Editora Nacional, 1966, p.244.

36. L'expression a été forgée par José López Rubio en référence à la célèbre « Génération de 27 », qui regroupe essentiellement des poètes, parmi lesquels figurent Federico García Lorca, Rafael Alberti, Vicente Aleixandre, ou encore Luis Cernuda, pour ne citer que quelques-uns d'entre eux. José López Rubio, Discurso de ingreso en la Real Academia Española [Discours d'entrée àl'Académie Royale Espagnole], Madrid, 1983.

\section{RÉSUMÉS}

Fondée sur une esthétique de la répétition, voire du ressassement perpétuel, la littérature populaire de masse constitue une forme de "prêt-à-lire » à destination d'un lecteur considéré avant tout comme un consommateur. A l'intérieur de cette production, le roman d'amour apparaît comme un genre particulièrement codifié, qui s'organise autour de motifs récurrents et de séquences narratives et thématiques stéréotypées. Dans sa trilogie romanesque, qu'il définit d'emblée comme une satire littéraire, Enrique Jardiel Poncela propose au lecteur une promenade ludique et démystifiante dans les coulisses du genre. Rédigée à la fin des années 1920, l'œuvre de l'écrivain madrilène s'inscrit, en effet, dans un contexte de méfiance généralisée à l'égard des stéréotypes, caractéristique des Avant-gardes littéraires et artistiques. Outre la dénonciation directe par le biais de commentaires métanarratifs particulièrement caustiques, la dimension 
iconoclaste de la trilogie apparaît sous la forme d'une dénudation systématique et ironique des paradigmes narratifs traditionnels. La séquence stéréotypée du fameux duo d'amour, avec ses traits récurrents, tels que la promenade au clair de lune, le premier baiser ou les larmes de l'héroïne, fait ainsi les frais de l'humour anticonformiste et subversif de l'auteur. Toutefois, pour Jardiel Poncela, déconstruction n'est pas synonyme de destruction. A l'encontre des Dadaïstes qui prétendaient faire table rase des conventions dominantes, le jeune auteur, quant à lui, entend faire œuvre de création grâce à une récupération ludique et inventive du stéréotype. C'est ainsi que pour redynamiser des pratiques romanesques sclérosées, Jardiel Poncela se réfère non seulement à de nouveaux moyens d'expression, tels que la bande dessinée ou le cinéma, mais il sollicite également la coopération d'un lecteur complice qu'il associe en permanence à ses jeux de déconstruction et de reconstruction. La subversion du stéréotype mène ainsi à la mise en oeuvre de nouvelles pratiques narratives qui inscrivent la trilogie dans le vaste mouvement de rénovation de l'écriture romanesque qui marque le début du XXe siècle.

Basada en una estética de la repetición rayana a veces en lo machacón, la literatura popular de difusión masiva programa una lectura formateada destinada a un público considerado como mero consumidor. Dentro de esta producción, la novela de amor aparece como un género sumamente codificado que se estructura en torno a una serie de motivos recurrentes y de secuencias (narrativas y temáticas) estereotipadas. En su trilogía novelesca, definida de entrada como una sátira literaria, Enrique Jardie Poncela invita al lector a dar un paseo lúdico y desmitificador por los caminos trillados del género. Redactada a finales de los años 20, la obra del escritor madrileño entra, en efecto, en el marco de las vanguardias literarias y artísticas que se caracterizan por una desconfianza absoluta con respecto a los estereotipos. Además de la denuncia directa mediante una serie de comentarios metanarrativos mordaces, la dimensión iconoclasta de la trilogía se trasparenta en la ostentación, sistemática a la par que irónica, de los paradigmas narrativos tradicionales. De esta forma, la secuencia estereotipada del consabido "dúo de amor ", con sus motivos recurrentes, tales como el paseo bajo la luna, el primer beso o las lágrimas de la joven enamorada, sufre la acción disolvente del humor anticonformista y subversivo del autor. Sin embargo, para Jardiel Poncela, «deconstrucción » no significa "destrucción». A diferencia de los dadaístas que aspiraban a hacer tabla rasa de las convenciones dominantes, el autor novel se propone crear una obra de arte mediante la recuperación lúdica e inventiva del estereotipo. Así, para vivificar unos procedimientos narrativos totalmente estancados, Jardiel Poncela no sólo se refiere a nuevos medios expresivos, tales como el cómic o el cine, sino que pide también la colaboración activa de un lector cómplice al que asocia constantemente a sus juegos de deconstrucción y reconstrucción. La subversión del estereotipo lleva por consiguiente a la elaboración de una nueva preceptiva narrativa que enmarca a la trilogía en el amplio movimiento de renovación de la escritura novelesca de principios del siglo XX.

\section{INDEX}

Mots-clés : réception, stéréotypes narratifs, Avant-gardes, subversion, écriture romanesque, Enrique Jardiel Poncela

\section{AUTEUR}

\section{CÉCILE FRANÇOIS}

Maître de conférences à l'Université d'Orléans, « META 